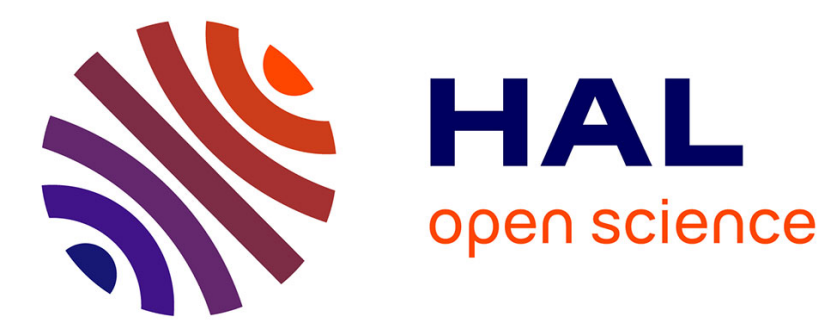

\title{
L'émancipation coopérative face à la compétence participative
}

Patrick Gaboriau

\section{To cite this version:}

Patrick Gaboriau. L'émancipation coopérative face à la compétence participative. L'émancipation coopérative face à la compétence participative, 2012. hal-00710755

\section{HAL Id: hal-00710755 \\ https://hal.science/hal-00710755}

Submitted on 13 Apr 2015

HAL is a multi-disciplinary open access archive for the deposit and dissemination of scientific research documents, whether they are published or not. The documents may come from teaching and research institutions in France or abroad, or from public or private research centers.
L'archive ouverte pluridisciplinaire HAL, est destinée au dépôt et à la diffusion de documents scientifiques de niveau recherche, publiés ou non, émanant des établissements d'enseignement et de recherche français ou étrangers, des laboratoires publics ou privés. 
VOL. 32, $\mathrm{N}^{\circ} 1,2013$

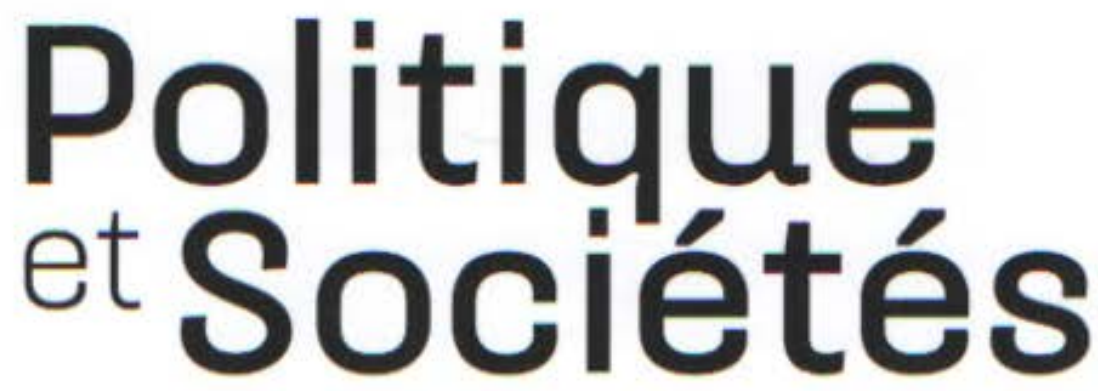

La revue de la Société québécoise de science politique

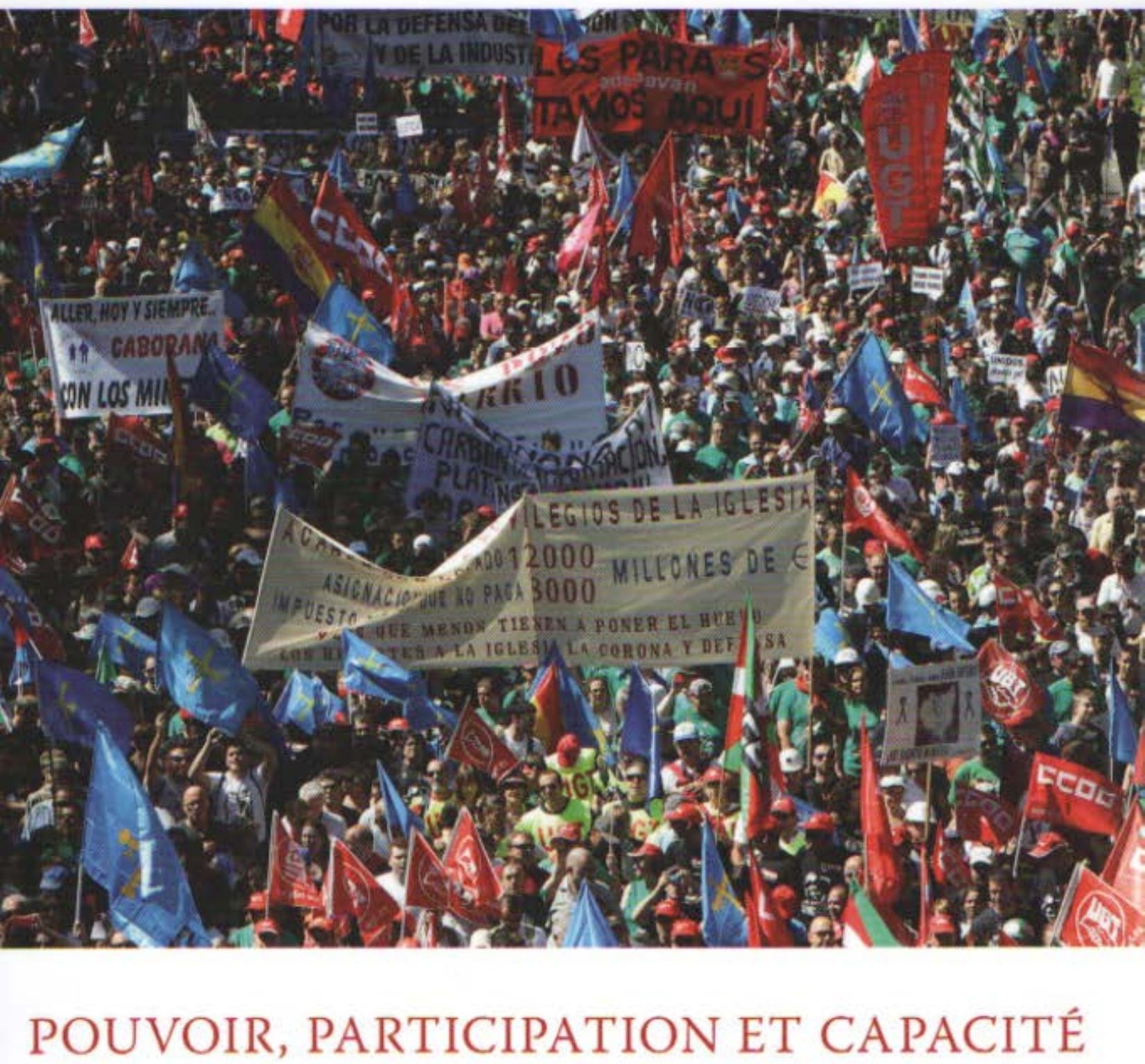




\title{
L'émancipation coopérative face à la compétence participative
}

\author{
Jérôme Boissonade \\ Université du Littoral (ULCO, France) \\ jboisson@msh-paris.fr
}

Nous souhaitons interroger la pertinence des notions de compétences et de capacités comme concepts critiques pragmatistes opératoires face aux nouveaux processus de domination, notamment ceux exercés par les institutions. Pour ce faire, nous nous appuyons dans ce texte sur l'expérience croisée d'un conseil de quartier et d'une accession sociale à la propriété à gestion coopérative. La démocratie participative exige des capacités spécifiques qui sont en effet perturbées par cette expérience coopérative dont le rapport à la décision repose sur le faire et la dimension capacitaire sur les prises élaborées à cet effet. Il s'agit donc moins pour les accédants concernés d'obtenir une reconnaissance publique de capacités pouvant conduire à un accès à la décision, que de construire de manière coopérative des prises d'émancipation critique vis-à-vis de ces dispositifs participatifs. Ce passage d'une compétence participative à une émancipation coopérative permet à la fois de surmonter l'instrumentalisation capacitaire et de mieux travailler l'articulation entre la constitution des prises et la portée critique des processus d'émancipation.

Cooperative Emancipation Facing Participative Expertise - We would like to examine how effective the ideas of skills and capacities are as operative pragmatic critical concepts in the light of the new processes of domination, particularly those exerted by institutions. To accomplish this, we have based our investigation in this text on the joint experience of a local council and the social accession to the ownership of a jointly-managed property. We find that the specific capacities required by participatory democracy are disrupted by this cooperative experience: the decision-making processes rely on actions and the capacity dimension on the grips designed to that effect. Those concerned by social accession therefore do not seek public recognition of capacities that could lead to some influence in the decision so much as the joint creation of critical emancipation grips with regards to these participative processes. This movement from participative expertise to cooperative emancipation helps to overcome the compulsion of competences and improve the links between the elaboration of the grips and the critical impact of emancipation processes. 
Les réflexions sur les dispositifs participatifs s'interrogent le plus souvent sur le sens accordé à la participation au sein d'une démocratie représentative et de processus décisionnels marqués eux aussi par l'idéal délibératif ${ }^{1}$. Qu'est-ce qu'un dispositif coopératif peut nous apprendre sur les capacités d'action et les engagements politiques des citadins, qui nous permettent de dépasser un face-à-face en trompe-l'œil entre participation et représentation? Plutôt qu'une analyse comparative de ces deux types d'agencement, cette contribution souhaite, par le biais de cette expérience coopérative, mettre à l'épreuve deux hypothèses.

Ceux qui participent s'efforcent le plus souvent d'obtenir une reconnaissance institutionnelle de leurs compétences, afin que les gouvernants leur accordent un pouvoir de décision accru. Pourtant, nous faisons l'hypothèse que le lien entre participation et décision dépend d'abord d'un registre particulier d'émancipation critique des dominés. Cette émancipation critique relève selon nous d'une défiance constructive qui ne repose pas tant sur le dire critique des gouvernés, que sur la production et la réactualisation d'un faire démocratique alternatif, susceptible de contraindre «la collectivité à se mettre en permanence à l'épreuve d'elle-même» (Rosanvallon, 2006: 121).

La deuxième hypothèse part du constat que le management participatif s'est aujourd'hui emparé des termes «capacité» et «compétence». Alors que la sociologie pragmatiste et la sociologie urbaine usent de ces deux concepts pour mettre en évidence et comprendre le sens critique des engagements des citadins, ces notions ne semblent plus appropriées pour saisir les nouvelles formes de domination que subissent les gouvernés.

Pour interroger ces deux hypothèses, nous nous sommes appuyé sur un long travail socio-ethnographique, croisant les histoires d'un conseil de quartier et d'une accession sociale à la propriété lancée dans le cadre d'une opération d'éradication de l'insalubrité.

Après un rapide cadrage sur ce que permet une telle démarche de recherche et ce qu'elle complique quand il s'agit de penser l'émancipation et de critiquer les limites du modèle capacitaire, nous tenterons de comprendre dans une première partie comment une copropriété coopérative est susceptible de produire des épreuves et des "prises $^{2}{ }$ dont la portée politique dépasse la défense d'un simple intérêt patrimonial. Les mises à l'épreuve suscitées par cette accession sociale à la copropriété coopérative ont en effet

1. Dans une démarche de démocratie délibérative, les arguments doivent relever d'un échange d'informations ordonnées, de «bonnes raisons» formulées en termes de bien public et exprimées de façon «authentique et vraie», afin que la force du meilleur argument l'emporte au cours d'un processus politique ouvert (d'après Bächtiger et al., 2004, cité par Forsé et Lafaye, 2008: 196; voir aussi Blondiaux et Sintomer, 2002 et Blondiaux, 2008).

2. Une prise est la «rencontre entre un dispositif porté par la ou les personnes engagées dans l'épreuve et un réseau de corps fournissant des saillances, des plis, des interstices » (Bessy et Chateauraynaud, 1995: 239). 
produit des engagements inscrivant la réflexion des copropriétaires dans l'action et l'émancipation ${ }^{3}$.

Dans une deuxième partie, nous poursuivrons cette approche en montrant que la nature de cette émancipation procède pour l'essentiel d'un faire coopératif qui perturbe la démocratie participative dans son rapport singulier à la décision et à la question capacitaire. Cette mise à l'épreuve, entre faire coopératif et dire participatif, nous donnera alors l'occasion de réinterroger la pertinence pragmatique des concepts de compétences et de capacités pour comprendre et intervenir sur les nouveaux processus de domination institutionnels.

\section{Une expérience ethnographique}

Étant personnellement bénéficiaire d'une accession sociale à la propriété au même titre que 20 autres accédants, j'ai profité de cette rare occasion pour mener à bien l'enquête ethnographique particulière sur laquelle repose ce texte. Les nouveaux copropriétaires, provenant d'habitations à loyer modique (HLM), ont décidé contre toute attente, lors de leur arrivée dans les lieux, de doter la copropriété d'une gestion coopérative, au grand dam du vendeur, une société d'économie mixte (SEM). À peine installé, ce syndic coopératif a dû ensuite lancer une action en justice contre l'entreprise et la SEM qui avaient construit et vendu leur bien, en raison de la mauvaise réalisation du bâtiment commandité par la Mairie de Paris. À la suite de cette première mise à l'épreuve, ces nouveaux habitants ont remis en cause publiquement certaines décisions de la collectivité locale au sein et à l'extérieur du conseil de quartier.

Depuis mon emménagement, mon rôle de chercheur-participant a nécessité un travail de réflexivité constant pour «situer» (Conein et Jacopin, 1994: 488) un engagement qui devait être porté par les décisions collectives, tout en participant à ouvrir des perspectives face aux processus d'imposition et de clôture mis en œuvre par les acteurs urbains et institutionnels ${ }^{4}$. Cette position va s'avérer essentielle par la suite, mais elle reste toujours délicate ${ }^{5}$. Toute la difficulté est d'éviter de produire un «enclicage ${ }^{6} »$, tout en mettant

3. 'Dans une conception pragmatique, les motifs sont considérés comme «des interprétations qui sont validées dans la mesure où elles introduisent de nouvelles perspectives et ouvrent de nouvelles possibilités d'action qui demandent elles-mêmes à être vérifiées par l'enquête démocratique» (Bohman, 2008: 18).

4. Voir à ce sujet le beau travail de Mathieu Berger (2009:15) sur les interactions politiques en public dans le cadre «d'asymétries justes».

5. Les questions épistémologiques, éthiques et pratiques que soulève cette recherche feront l'objet d'un autre article centré sur les enjeux que pose cette démarche croisant réflexion ethnographique et recherche action.

6. Habituellement, «le recours à un interprète, qui est toujours aussi un 'informateur privilégié', introduit des formes particulières d"enclicage': le chercheur dépend alors des 
en œuvre lors des situations un «pluralisme ethnographique de l'ordre de la socialisation de la logique de l'enquête» (Pierce, cité par Cefaï, 2010 : 561) susceptible de resymétriser, autant que faire se peut, la distribution inégale des prises lors des épreuves. Cette posture de recherche assume une certaine « impatience» (Stavo-Debauge, 2011) qui m'a conduit à réinjecter mes bricolages interprétatifs dans les situations sans réserver l'imagination d'alternatives pour d'autres scènes, une fois la validation académique effectuée ${ }^{7}$. La mise à l'épreuve, et donc la validation, de ces bricolages interprétatifs dépend donc, d'une part, du déplacement des situations qu'ils peuvent entraîner. D'autre part, cette validation dépend de la capacité de ces bricolages à amenuiser le caractère asymétrique de ces situations. C'est par exemple cette méthode qui a été utilisée lors du processus qui a guidé mon observation du conseil de quartier auquel participaient plusieurs copropriétaires ${ }^{8}$. Cette démarche de recherche doit permettre, d'une part, de penser les compétences en termes de prises instituées, élaborées ou conquises de manière situationnelle, pragmatique et pluraliste et, d'autre part, de comprendre les mobilisations en termes d'émancipations qui s'appuient sur ces prises et qu'elles participent à façonner ou au contraire à déconstruire dans des visées tactiques ou stratégiques. Ces situations co-construites avec le chercheur participaient donc d'une tentative de resymétrisation et de pluralisation des perspectives, mais nous verrons qu'elles ont surtout été appropriées par les acteurs dans un processus herméneutique au sein duquel les dimensions coopératives et publiques ont joué un rôle essentiel.

propres affinités et hostilités de son interprète, comme des appartenances ou des ostracismes auxquels le voue le statut de ce dernier» (Olivier de Sardan, 1995: 101). Pour notre terrain, il s'agit du rapport inverse dans lequel ce sont les acteurs qui peuvent dépendre du chercheur.

7. Bruno Latour (1995) différencie la recherche, en train de se faire, incertaine, de la science, publiée, reconnue par les instances académiques.

8. Indépendamment de toute décision collective, quelques copropriétaires, six mois après leur emménagement, ont en effet posé leur candidature et certains ont été retenus pour participer au conseil de quartier. Inventés dans les années 1970, les conseils de quartier sont des dispositifs consultatifs qui ont été institués par la loi du 27 février 2002, dite Loi Vaillant, relative à la démocratie de proximité. Si leur existence est rendue obligatoire pour les communes de plus de 80000 habitants, leur forme est laissée à l'appréciation des collectivités. Certains conseils de quartier fonctionnent en réunions publiques associant ceux qui le souhaitent sans ordre du jour figé. Celui qui nous intéresse est composé de deux collèges, associatif et habitants. Pour ce deuxième collège, les conseillers de quartier sont nommés par tirage au sort (voir sur cette problématique Sintomer, 2007). Son fonctionnement et ses prérogatives consultatives sont précisés par une charte d'arrondissement. Il se réunit en général en conseil restreint, sauf lors de rares réunions publiques. Sa présidence est assurée par un maire adjoint et deux vice-présidents provenant du collège associatif, inamovibles de fait (le collège associatif est en termes de présence effective le plus nombreux et il est composé de membres cooptés). Ceux-ci préparent les ordres du jour et dirigent les débats, relatifs le plus souvent à l'agenda municipal, plus qu'aux préoccupations exprimées par les conseillers habitants. 


\section{Les prises de l'émancipation}

\section{Mises à l'épreuve}

Revenons tout d'abord sur les deux mises à l'épreuve de la copropriété coopérative qui l'ont opposée au constructeur et au vendeur du bâtiment, puis à la Mairie d'arrondissement. La dimension sérielle (Quéré, 2009: 316) de ces épreuves a produit un réel travail d'apprentissage lors duquel se sont exercés des engagements. Des prises sont nées à ces occasions, insoupçonnées parce que distribuées dans les situations qui les avaient révélées.

La première mise à l'épreuve qui mobilise la quasi-totalité des copropriétaires constitués en coopérative les oppose au constructeur et au vendeur. Après s'être dotés d'un syndic coopératif, les accédants émettent en effet des réserves sur la bonne réalisation des ouvrages (parties communes et privatives), d'abord mentionnées de vive voix lors des visites de livraison, puis par courriels, courriers avec accusé de réception et enfin par une assignation au tribunal de grande instance. La conjonction de plusieurs facteurs avait en effet abouti à un suivi et a un état du chantier médiocre, ainsi qu'à de nombreuses malfaçons une fois le bâtiment livré. Attribué à un public de locataires de HLM ou géré par un syndic privé, celui-ci n'aurait pas souffert de contestation généralisée. Ici, malgré la levée officielle des réserves par la SEM, les copropriétaires vont rapidement rassembler leurs griefs, en mesurer souvent la portée générale et construire progressivement un bilan précis opposable aux différentes parties prenantes. Dans le rapport de force qui s'engage, le poids des différents acteurs paraît pourtant inégal. D'un côté, une multinationale du bâtiment, possédant seule la compétence pour juger de la qualité technique de ce qui a été livré, ainsi que la Mairie de Paris, représentée par une société d'économie mixte composée essentiellement de juristes. De l'autre coté, 27 copropriétaires, tous ou presque inexpérimentés sur ces questions ${ }^{9}$ et représentant autant de situations personnelles en termes de ressources financières, de parcours résidentiel ou de personnalité ${ }^{10}$. Structurés par le syndic coopératif constitué comme porte-parole, y compris pour des dommages privés, ils restent pourtant a priori difficiles à mobiliser dans une démarche juridique demandant au préalable un engagement financier non négligeable. Pour rééquilibrer ce rapport de force, le syndic

9. À l'exception notable du chercheur lui-même, ancien architecte.

10. La diversité des montages financiers a conduit à une homogénéité socioprofessionnelle moins grande qu'il n'y paraît et les situations financières sont très contrastées. Une diversité que l'on trouve aussi sur le plan des profils culturels (notamment Maghreb et Afrique noire). C'est donc plutôt cette diversité qui tranche avec l'homogénéité relative du quartier en termes de visibilité sociale et ethnique, majoritairement nord-africaine. Par ailleurs, hormis un responsable syndical actif (non permanent), on ne trouve pas d'ancrage partisan notable parmi les copropriétaires. Ce que montre la dizaine d'entretiens réalisés jusqu'à maintenant parmi les copropriétaires, c'est plutôt la capacité de cette expérience coopérative à stimuler des engagements inédits jusqu'alors chez ces personnes. 
coopératif va devoir trouver les montages financiers convenables et surtout construire la légitimité des dommages en question. Par un travail d'objectivation technique et juridique ${ }^{11}$, les copropriétaires s'instituent non sans difficulté en interlocuteurs légitimes vis-à-vis des autres acteurs. Le ressenti des problèmes d'isolation phonique, des travaux inachevés, des infiltrations ou le vertige que l'on peut éprouver dans un escalier sur deux niveaux sont en effet difficiles à dire. Leur format n'étant pas préétabli, ces épreuves sont considérées comme subjectives. Plaidant la normalité des ouvrages, l'entreprise va opposer un déni quasi systématique à l'expérience subjective de chacun. Cependant, la plainte n'est plus portée par un ou des copropriétaires, mais par un porte-parole coopératif, une personne morale qui s'attache à dépersonnaliser la relation de chacun avec le vendeur-constructeur pour considérer des malfaçons partagées, puis la repersonnalise en publicisant les préjudices, voire les souffrances subies par les personnes. Cette volonté de dépersonnalisation/repersonnalisation des relations nécessite pour le syndic un travail de qualification mettant en relation les problèmes contextuels rencontrés sur ces habitations et des figures symboliques (politiques et morales) susceptibles de les exprimer. Ce faisant, les copropriétaires transforment ces «épreuves existentielles» en «épreuves de réalité» (Boltanski, 2008a) et peuvent faire appel aux élus (maire et adjoints au maire de Paris et leurs homologues de l'arrondissement, député de la circonscription) en ayant qualifié et donc attribué une valeur publique aux situations sociospatiales domestiques qu'ils rencontraient, afin de proposer une réalité concurrente aux récits déployés par l'entreprise et la SEM, cette dernière étant pourtant missionnée par la Ville de Paris ${ }^{12}$.

La deuxième mise à l'épreuve de la copropriété coopérative va l'opposer à la Mairie d'arrondissement. Le mode de fonctionnement de ce dispositif consultatif qu'est le conseil de quartier tranche en effet radicalement avec celui qui caractérise le syndic coopératif. Non seulement cette assemblée qu'est le conseil de quartier est découplée de l'action et de la décision, mais elle relève aussi le plus souvent d'une instrumentalisation dans laquelle l'adjoint au maire de Paris en charge de la démocratie locale et de la vie

11. Cette mise en forme a d'abord consisté à compiler l'ensemble des griefs rapportés par les copropriétaires. Il a fallu ensuite décrire ces griefs non pas en termes de préjudice produit, mais de défaut de construction, puis mettre en équivalence les préjudices comparables. Enfin, un partage a été opéré entre les réserves concernant les parties communes et celles touchant des parties privatives. Le tout a donné lieu à la rédaction d'un «Rapport technique » à laquelle j'ai participé, qui a ensuite été intégré à une assignation au tribunal de grande instance de Paris. Une sélection de ces dommages a aussi été déclarée à l'assurance dite de «dommagesouvrages ». Les sommes en jeu dépassent plusieurs centaines de milliers d'euros.

12. Une telle conception des mobilisations rejette la coupure habituelle entre les pratiques sociales ordinaires et les formes d'action collective à caractère politique, pour privilégier «les continuités et les relations complexes qui se nouent entre les deux ensembles de pratiques ainsi distingués et opposés» (Bonny et al., 2012: 8). 
associative considère qu'«il faut faire des citoyens, des outils de la politique de participation $»^{13}$. Une conception de la participation relayée à de multiples reprises par le président du conseil de quartier, adjoint au maire: «L'objectif, demain à la réunion publique, c'est qu'un maximum de personnes s'expriment [...] C'est là où les gens du quartier vous voient, c'est important [...] Lorsque vous vous exprimerez demain, dites bien que vous êtes conseillers de quartier, c'est important ${ }^{14}$.» "Pour les réunions publiques je serai là, mais je préfèrerais que ce soit vous qui animiez. Ce serait une direction collégiale ${ }^{15}$.»

La démocratie locale se résume ici à une «scène paradoxale qui fait voir la contradiction des deux logiques, celle de la force des arguments que partage la 'communauté débattante' et celle du rapport de forces qui structure la décision politique» (Fourniau, 2007: 174). Les discussions et les quelques résolutions qui émaillent les conseils de quartier restreints auxquels je participe sont en effet essentiellement destinées à donner une légitimité aux décisions du politique, de plus en plus susceptibles aujourd'hui d'être contestées. Ici,

la force n'est pas limitée par l'exigence de légitimité, elle se l'adjoint. Cette adjonction ne peut pas être élaborée abstraitement, elle s'incarne dans des dispositifs techniques et juridiques concrets, dans des accomplissements problématiques, qui alors emportent l'accord du plus grand nombre et sont jugés, par conséquent, légitimes. (Linhardt et Moreau de Bellaing, 2005: 297)

Face à ces pratiques, les conseillers de quartier ont de réelles difficultés à porter une parole crédible, notamment lors des réunions restreintes aux seuls membres du conseil. D’une part, la possibilité de revendiquer un engagement partisan (parti politique, syndicat...) leur est déniée puisqu'ils doivent «s'abstenir de tout prosélytisme ${ }^{16}$ ». D'autre part, leur absence de représentativité due à leur nomination par tirage au sort particularise toute prise de position et rend ambigu un travail de montée en généralité ${ }^{17}$.

13. Propos tenus lors du Printemps de la démocratie locale, le 4 avril 2009.

14. Conseil de quartier restreint, réunion du 10 février 2009.

15. Président du conseil de quartier, adjoint au maire, chargé de la citoyenneté et de l'accompagnement scolaire, Conseil de quartier restreint, 5 janvier 2009.

16. Article 13 de la Charte des conseils de quartier de l'arrondissement.

17. Cette double injonction de non-ancrage contraste avec les pratiques des habitants lors des réunions publiques. De manière quasi systématique, ces derniers commencent en effet leur intervention en indiquant leur degré d'ancienneté et leur lieu de résidence exact dans le quartier pour étayer leurs dires, puis tentent de faire partager ce qui est dit, le plus souvent en référence à un principe de «commune humanité» (Boltanski et Thévenot, 1991), par exemple lorsqu'un artisan au chômage qui cherche un local d'activité dans le quartier conclut sa prise de parole par: «Je voudrais lutter contre l'exil», ou lorsqu'un intervenant se déclare explicitement comme porte-parole: «Je suis le marchand de journaux. Je représente la communauté musulmane et nous demandons un local pour faire notre prière dignement. » Réunion publique, 17 mars 2009. 
Fragilisés dans leur capacité à exploiter la première possibilité de montée en généralité (non-engagement) et privés de la deuxième (non-représentativité), les copropriétaires-conseillers de quartier se sont appuyés sur leurs expériences habitantes partagées et leur démarche coopérative pour produire des solutions alternatives aux positions défendues par l'institution municipale (sens de circulation, piétonnisation de rue, «quartier tranquille», etc.) et au fonctionnement participatif du conseil de quartier (statut et rôle du président, ordre du jour, autonomie, etc.). Cette démarche critique sur la forme et le contenu de la démocratie participative nécessitait cependant pour les copropriétaires-conseillers de quartier de dénouer les relations entre chaque habitant ou conseiller et l'adjoint au maire et/ou président de conseil de quartier au sein de chaque enceinte participative (conseil de quartier, réunion publique de concertation, etc.), afin d'établir et de rendre prépondérantes les relations entre ces habitants ou conseillers et les expériences qu'ils pouvaient partager au quotidien. Au sein du conseil de quartier, la démarche des copropriétaires a abouti à l'élaboration d'un manifeste dont les conclusions ont été nourries en grande partie par l'expérience coopérative de la décision et un rapport que j'avais rédigé auparavant, à la demande du président du conseil de quartier. Ce manifeste ainsi que le rapport semblent avoir contribué à transformer le fonctionnement du conseil de quartier ${ }^{18}$, voire ceux de l'ensemble de l'arrondissement ${ }^{19}$. Une telle issue provisoire semble donc confirmer la capacité de ce dispositif coopératif à porter la critique au sein de cet autre dispositif, participatif celui-là, qu'est le conseil de quartier. Contrairement au caractère consultatif de ce dernier, l'expérience coopérative consiste en effet, nous allons le voir, à apprendre, à juger et à agir conjointement en regard des conséquences escomptées, bref, à considérer le réel à travers l'action que l'on peut déployer ensemble pour le transformer. Envisager ainsi la connaissance comme pratique des conséquences, c'est mettre en évidence son rôle dans les processus d'émancipation qui sont à l'œuvre.

\section{Émancipations}

La démarche judiciaire engagée contre le vendeur-constructeur du bâtiment témoignait d'un premier travail d'émancipation critique lors duquel les copropriétaires ont dû réfuter l'accusation, portée par la SEM et l'entreprise,

18. Le manifeste s'intitule L'organisation et la fonction du conseil de quartier en 13 questions. Il porte sur le rôle du président du conseil, la proportion de conseils restreints par rapport à ceux publics, le caractère uniquement consultatif des décisions, la détermination de l'ordre du jour, la représentativité des associations, la désignation des associations, le vote des décisions, les comptes rendus, la communication interne, le bureau du conseil, le rôle des conseillers, le rôle des suppléants et l'utilisation des budgets des conseils de quartier.

19. Le tirage au sort et les conseils de quartier restreints viennent d'être supprimés pour l'ensemble de l'arrondissement, au profit de réunions publiques et de commissions auxquelles chacun peut participer sans restriction. 
de démesure de «l'action en justice» lancée à leur encontre. Le manifeste relevait d'un deuxième effort d'émancipation, cette fois vis-à-vis des cadres participatifs imposés par l'institution municipale. Il semble que cette double épreuve que les copropriétaires ont eu à affronter leur a permis, lors d'une troisième "affaire», d'associer force et légitimité face à celles déployées par l'institution municipale et de mieux résister à l'assignation au rôle stigmatisé du riverain NIMBY (not in my backyard) (Jobert, 1998). L'action engagée à propos de la piétonnisation des rues mitoyennes va constituer, en effet, le dernier mouvement à ce jour de cette émancipation.

Les travaux de rénovation urbaine qui ont conduit à la réalisation de cet immeuble relèvent pour la Mairie de l'arrondissement d'un «urbanisme qui vient se substituer aux problèmes du quartier $»^{20}$. Les enjeux dépassent donc clairement la bonne réalisation du bâtiment, puisque l'objectif que remplit ce dernier est d'abord d'effacer «ce qu'il en est de ce qui était» auparavant (Boltanski, 2008a), qu'il s'agisse de l'élimination des squats, de lutte contre la toxicomanie ou la prostitution. Pour la collectivité, ce projet vaut moins pour ce qu'il procure à ses habitants que pour l'effet durable qu'il produit sur son milieu.

Les dimensions publiques données au projet urbain vont fournir un appui paradoxal, mais essentiel, au travail d'émancipation des accédants. D'un côté, chaque copropriétaire a dû construire son rôle dans ce contexte au sein duquel l'institution municipale souhaite qu'une nouvelle appropriation chasse l'ancienne ${ }^{21}$ afin de garantir dans le temps la transformation socio-urbaine du quartier. De l'autre, chacun constate que ce bâtiment possède une dimension à la fois individuelle (patrimoniale), collective (copropriété coopérative) et publique (accession sociale aidée) qui donne à ces habitants, acteurs au quotidien du projet urbain d'éradication de l'insalubrité, une légitimité particulière pour accéder à la décision. La démarche adoptée par les copropriétaires va donc immédiatement anticiper l'accusation de privilégier l'intérêt particulier sur l'intérêt général. Une pétition, ainsi que plusieurs courriers et courriels vont être envoyés au maire de l'arrondissement et à l'adjoint responsable de la voirie. Des entretiens auront lieu avec ce dernier et avec d'autres adjoints. Les risques d'accident entre les véhicules et les piétons, notamment les enfants des copropriétaires, qui sont nombreux à évoluer dans cette rue au statut incertain, sont régulièrement mentionnés par les copropriétaires, mais c'est aussi le souci de l'autre qui les motive: «On ne veut pas fermer la rue pour être entre nous, mais au

20. Maire de l'arrondissement indiquant que la rénovation des immeubles sécurise le quartier, Compte rendu de mandat, 16 juin 2009.

21. Cette phase critique préalable à l'emménagement des copropriétaires dans ce quartier a fait l'objet d'un ouvrage ethnographique d'Isabelle Coutant (2000). Nous consacrerons sans doute un texte ultérieur à notre « revisite» circonstancielle (Buravoy, 2010). 
contraire l'ouvrir aux piétons de l'extérieur ${ }^{22}$.» Autrement dit, d'un côté la collectivité élabore un projet urbain reposant de manière tacite ou explicite sur une coveillance (Jacobs, 1961; Wekerlé, 1999) et un effet NIMBY, pour garantir une transformation durable de la population fréquentant cet espace; de l'autre, les copropriétaires doivent dénier toute intention de ce type afin de rendre légitime leur action face à une collectivité susceptible de le leur reprocher.

La réfutation de tout comportement NIMBY (Trom, 1999) par les copropriétaires va donc représenter un véritable travail d'émancipation vis-à-vis de leur seule condition de propriétaire d'un bien privé, mais aussi vis-à-vis des paradoxes de l'institution qui, tacitement, les a affublés de leur nouvelle condition spatiale et statutaire. Cette émancipation a été rendue possible parce que les copropriétaires se sont approprié leur pratique coopérative (gestion du bâtiment, procédure judiciaire, courriers, présence sur place lors des fêtes et des actions collectives, etc.) comme constitutive d'une morale identitaire politique alternative qui a permis à chacun d'assumer et de défendre l'engagement collectif ${ }^{23}$ dans ses conséquences publiques (réalisation et diffusion de pétitions, rendez-vous en Mairie, courriers, présence active aux réunions publiques, fabrication d'affiches, participation au conseil de quartier, etc.).

\section{Le dire et le faire}

Cet engagement coopératif à la visée émancipatrice contraste avec une démocratie participative qui s'inscrit aujourd'hui dans une injonction contradictoire à «l'efficience ${ }^{24} »$. On demande au conseiller de quartier de s'intéresser aux affaires publiques et d'y participer, de posséder une personnalité prenant en compte les intérêts de la communauté et les principes démocratiques, de percevoir correctement les réalités politiques et d'en débattre, pour, enfin, juger de façon rationnelle (Berelson, 1952: 329; Lagroye, 1991: 310). Tiré au sort, chaque conseiller de quartier ne peut parler qu'en son nom, mais se doit aussi «d'avoir une vision pour le quartier ${ }^{25}$ ». Cette injonction démiurgique est un prérequis implicite que doit présenter le conseiller afin

22. Présidente du syndic coopératif et conseillère de quartier, Réunion de copropriétaires du syndic coopératif avec le conseiller municipal de l'arrondissement et conseiller de Paris délégué aux Quartiers verts et Paris respire, Mairie de l'arrondissement, 10 septembre 2009.

23. Lors de l'ultime réunion publique consacrée au projet d'aménagement de la rue, le public présent est arrivé à imposer, à l'adjoint présent, l'examen par la Préfecture d'un projet alternatif soumis auparavant par un groupe de copropriétaires, dont le chercheur. Ce projet a finalement été partiellement mis en œuvre, en lieu et place du projet municipal.

24. «Si la précédente mandature a posé le cadre de la politique participative, la deuxième doit reposer sur l'efficience.» Chef de la Mission de démocratie locale de la Ville de Paris, Rencontres de la démocratie locale de l'arrondissement, 14 mars 2009.

25. Président du conseil de quartier, entretien du 7 juillet 2009. 
que son expertise soit reconnue. Pourtant, d'une part, seule la reconnaissance des autres permet d'accéder au général (Boltanski, 1984: 40). D'autre part, «les compétences en vue de l'action sont disponibles sur un mode relatif, car leur engagement dépend étroitement du contexte situationnel. Elles sont également disponibles sur un mode relatif en ce sens qu'elles sont inégalement distribuées ${ }^{26}$.» Si cette capacité relative «vit et meurt en interaction» (Munck et Zimmermann, 2008: 14), on ne peut donc demander à chaque conseiller d'appréhender clairement une «vision» avant même que le conseil de quartier ne puisse atteindre, s'il le peut, ce but commun, si tant est qu'il soit légitime.

Parallèlement, l'objectif d'efficience de l'action publique participative a conduit la Mairie de Paris à proposer une offre de formations destinées aux conseillers de quartier. Celles-ci ont pour but de familiariser les conseillers au fonctionnement de la ville, de leur donner les moyens de «bâtir et de réaliser leurs projets» et enfin de les aider «à informer [et à] rendre compte de leur activité, mais aussi à développer des échanges inter-habitants $»^{27}$. Il s'agit donc d'un corpus de formation consacré essentiellement à l'acquisition d'un savoir-faire et d'un faire savoir, susceptibles de rendre le conseiller compétent sur les scènes où il doit agir et dans le rôle qui lui a été attribué par l'institution. Face aux experts (élus, techniciens... ), le profane devient alors compétent, mais, utilisant les mêmes outils sémantiques, il réduit à son tour les grammaires utilisées dans le débat public à celles portées par les institutions. Le mouvement gestionnaire en cours perceptible dans cette démarche conduit à fixer,

par des signes et des images ad hoc, la dénotation des situations et d'en faire système [...], à créer et à imposer des formes langagières s'appuyant sur la seule valeur expressive des mots en shuntant leur valeur affective, idéologique ou éthique [...] Sur ces bases peut alors se développer une pensée vectorielle et séquentialisée, peu compréhensive et acritique de l'action. (Chauvière, 2007: 169)

L'ambition capacitaire menée par l'institution municipale semble donc s'accompagner d'une euphémisation des motifs et des raisons de l'action au profit du vocabulaire participatif.

$\mathrm{Au}$ sein du conseil de quartier, le dire détermine la «grandeur» du conseiller. $\mathrm{Si}$ «les corps ne peuvent exprimer leurs qualités qu'au travers des dispositifs» (Bessy et Chateauraynaud, 1995: 245), c'est ici sa capacité rhétorique qui est déterminante, dans une enceinte privilégiant le moment

26. Danny Trom (1997: 107), en référence à la notion de «disponibilité relative» de Nicolas Dodier et Isabelle Baszanger (1997: 54).

27. Ces principes généraux sont ensuite déclinés arrondissement par arrondissement. Consulté sur internet (http://www.paris.fr/portail/viewPDFileServlet?file_id=8004) le 7 février 2010 . 
présent et la performance publique. En revanche, au sein de la copropriété coopérative, face aux situations problématiques rencontrées, c'est d'abord le faire qui qualifie ${ }^{28}$ ce que sont les uns ou les autres, chacun étant mis à l'épreuve des charges qu'il a "accepté» de prendre pour le collectif (Castoriadis, 1999: 118). La première arène, participative, du conseil de quartier se caractérise par l'inconséquence relative de délibérations qui ne sont pour la quasi-totalité que consultatives. En revanche, dans la deuxième, celle de la copropriété coopérative, le manquement individuel est lourd de conséquences sur chacun, chaque décision étant en mesure d'entraîner des problèmes matériels, voire des conflits interpersonnels importants. Dans ce faire, les corps sont mis en jeu de manière directe dans l'action collective, quasi quotidienne au moins pendant les deux premières années d'installation des accédants, mais aussi lors des événements ordinaires, qu'ils soient en lien avec la prostitution installée dans le quartier depuis de nombreuses années (appels au secours, disputes liées aux tractations, etc.), la circulation motorisée sur la rue piétonne mitoyenne, les agressions (tentatives de vol et vol de téléphones portables, de baladeurs...), les déchets (l'urine, les détritus...), les vols (vélos) ou même leur anticipation. D'où la difficulté à publiciser directement ces expériences dans lesquelles c'est l'intimité des corps qui est éprouvée. Cette publicisation des expériences rencontrées par les copropriétaires est difficile en raison d'un double soupçon d'irrationalité de la plainte et de non-efficience du citoyen. Si l'engagement coopératif a su partager ici l'intimité des expériences habitantes et émanciper ceux qu'il mobilisait, c'est semble-t-il parce qu'il s'appuie, d'une part, sur d'autres fondements que les intérêts croisés de chacun et, d'autre part, sur une conviction partagée de la nécessité d'agir en justice pour «socialiser l'injustice» (Boltanski, 2008b), conviction qui aboutira à la démarche judiciaire lancée contre la SEM et l'entreprise. Chacun a en effet inscrit cette action dans son parcours et sa situation. La fragmentation des expériences, qui paraissait au départ un handicap, s'est finalement révélée comme autant de prises contribuant à ce que l'action en justice soit stabilisée comme action juste pour chacun, que ce soit pour des raisons financières, politiques, symboliques, éthiques, etc. Cette justesse de l'action en justice est portée par des parcours individuels et effectuée par des ajustements réguliers lors du travail de gestion coopérative de la copropriété. Dans ce cadre, la responsabilité personnelle peut être jusqu'à un certain point assumée collectivement, de manière à pouvoir partager la culpabilité de l'échec et s'approprier la satisfaction de la réussite, sans pour autant s'être délesté de la décision, renvoyée à un ailleurs.

28. Pour John Dewey, la situation «est plutôt donnée, 'expériencée’ et constituée, de façon continue et soutenue, comme un contexte vécu et significatif, 'qualitatif et qualificatif' (1938: 70), d'interactions entre les humains et les choses» (Vandenberghe, 2009: 381). 


\section{Capacités}

Les prises qui se révèlent lors des différentes mises à l'épreuve que nous avons évoquées ne recoupent donc pas les compétences qu'appellent de leurs vœux les dispositifs participatifs. Par conséquent, il n'est pas surprenant que l'on puisse, grâce aux prises coopératives, maîtriser le faire au sein de la copropriété et ne pas se sentir compétent pour maîtriser le dire, en termes rhétorique et tactique, au sein du conseil de quartier. Jean-Louis Genard a montré dans quelle mesure la définition «capacitaire» de l'individu et du citoyen relève historiquement d'une anthropologie de la responsabilité (1999: 71-76) qui entremêle pouvoir et pouvoir sur soi (Cantelli et Genard, 2007 : 27). Mais peut-on faire reposer l'échec ou la réussite d'une participation au conseil de quartier sur les compétences que le conseiller a ou n'a pas, donc sur sa seule personne, sans s'interroger sur les limites du cadre rhétorique consultatif instauré par le conseil de quartier? Ou doit-on au contraire considérer qu'un dispositif public qui transforme ceux qui sont grands ailleurs, dans un autre contexte, en petits ici, au sein du conseil de quartier, manque à une de ses missions qui est de faire de tous des citoyens? Dans quelle mesure la mise à l'épreuve d'un tel dispositif de participation dans la ville interroge-t-elle le modèle capacitaire des sociologies pragmatistes et les limites de son appropriation par des combats politiques, des postures critiques et des logiques de reconnaissance?

De manière générale, on considère que l'individu possède une compétence majeure, celle qu'il a de sa langue et, au-delà, de tout un ensemble de règles et de principes qui constituent une grammaire qui structure ses relations. Cette compétence a progressivement été étendue à des domaines considérés jusqu'alors comme relevant de la sphère privée, pour leur donner toute leur dimension sociale. C'est notamment le cas des compétences pratiques et réflexives étudiées par Anthony Giddens (2000 : 43-50), du concept de «capital social» d'Antoine Bevort et Michel Lallement (2006) ou celui de « capabilité » développé par Amartya Sen, à la fois lié à l'individu, aux opportunités qui se présentent à lui et aux institutions qui sont dans le devoir de les garantir (De Munck et Zimmermann, 2008 : 15), ainsi que ceux de « compétence politique» de Loïc Blondiaux (2007), ou de compétence morale en termes de justice de Luc Boltanski et Laurent Thévenot (1991). La sociologie pragmatiste française s'est attachée tout au long des deux dernières décennies à élaborer un «modèle général de compétences des personnes» (Dodier 2001: 433, cité par Genard et Cantelli, 2008:2) qui sorte ces compétences de l'habitus et du calcul intéressé: Boltanski révèle «ce dont les gens sont capables » (1990), Thévenot observe «l'aptitude des acteurs [...], aptitude qui devrait occuper une place centrale dans une théorie de l'action» (1990: 40) et Isaac Joseph travaille les «compétences de rassemblement» (1996: 108). Cette démarche de recherche, orientée vers la reconnaissance des compétences des 
acteurs marginalisés, est aussi particulièrement prégnante en sociologie urbaine. Dès les années 1970, le droit à la ville (Lefebvre, 1968) et les luttes urbaines (Castells, 1975) théorisent les compétences citadines comme pouvoir d'appropriation. Dans les années 1980, les travaux d'Henri Raymond (1984) dotent les habitants d'une compétence pratique, celle de pouvoir agir sur l'espace; d'une compétence langagière, celle de pouvoir lui donner du sens en le qualifiant; et Michel de Certeau (1990) valorise quelques années plus tard les compétences d'interprétation et d'adaptation des acteurs. À partir de la fin des années 1990, la dimension capacitaire dans la recherche urbaine est notamment présente dans les analyses des processus participatifs liés aux projets urbains, dans les pays du Nord (Bacqué et Sintomer, 2011) et dans les pays du Sud (Berry-Chirkhaoui et Deboulet, 2000). Aujourd'hui, l'influence nord-américaine se fait plus présente avec une large discussion de la notion d'empowerment qui bute sur le soupçon communautaire et néolibéral, un obstacle que les discussions actuelles sur le community organizing tentent de surmonter. Le point commun de ces courants de recherche consiste néanmoins à attribuer in fine une qualité en propre à des individus dont la place au sein des processus socio-urbains est déniée par ceux qui les gouvernent: compétence de «l'habitant», du «citadin», de «l'expert du quotidien », etc.

Cependant, le management a repris à son compte cette dimension cognitive, réflexive et morale dans la gestion des organisations, notamment celles se revendiquant comme participatives. «L'enrôlement de la subjectivité » (Clot, 1995) effectué à partir de la fin des années 1970 s'est en effet progressivement étendu à tous les domaines pour aboutir à une valorisation, pleine d'ambiguïtés, des compétences dites personnelles. Le «savoir-être [est devenu] la compétence qui monte» (Wybo, Benhaeim et Feldman, 2010). Transposition d'un empowerment à la française (Genard et Cantelli, 2008: 10), cette injonction à la compétence apparaît à tous les échelons de la politique de la ville consacrée à la participation, du conseil de quartier qui demande aux conseillers d'agir «selon leurs compétences $»^{29}$, au Centre d'analyse stratégique ${ }^{30}$ qui enjoint de «développer les compétences ou tirer profit des talents des individus concernés» (2008: 56), en passant par la Mission démocratie locale qui en appelle aux «compétences pratiques » des habitants ${ }^{31}$.

29. «Tous les conseillers sont à égalité et agissent selon leurs compétences, motivations et disponibilités du moment. Chacun peut trouver sa place notamment au travers des commissions.» Compte rendu du conseil de quartier restreint, 20 septembre 2008.

30. Le Centre d'analyse stratégique est une institution d'expertise et d'aide à la décision auprès du premier ministre. Il a pour mission d'éclairer le gouvernement dans la définition et la mise en œuvre de ses orientations stratégiques en matière économique, sociale, environnementale ou technologique.

31. «L'usager, l'habitant, ont une expérience quotidienne de la ville. Pensez-vous qu'ils possèdent ainsi des compétences pratiques pouvant être sollicitées dans le cadre de la parti- 
Les différents dispositifs participatifs mis en place par la Mairie de Paris et leur évolution montrent que les concepts de sciences humaines façonnent de plus en plus ces dispositifs d'action publique (Nez, 2007; Busquet et al., 2010) ainsi que les outils sémantiques utilisés par les acteurs. La sociologie et la science politique devraient donc, selon nous, tirer les conséquences des évolutions de ces dispositifs non seulement en termes de critique de l'instrumentalisation de la recherche, liée au rôle d'expertise qu'on lui fait jouer, mais aussi et surtout en termes de redéfinition des concepts susceptibles d'opérer dans ces nouveaux contextes. Nous avons en effet signalé comment toute avancée de la recherche, toute critique sont susceptibles d'être exploitées par les gouvernants avant même qu'elles aient pu être appropriées par ceux que tous souhaitent mobiliser: les gouvernés. De fait, le concept de compétence contient une valeur opératoire devenue ambiguë. Il qualifie les capacités attachées aux individus ou aux collectifs, dans le but de faire reconnaître la place de ces publics marginalisés dans les jeux d'acteurs, cela alors que les nouveaux modes de gouvernance ont intégré cette capacitation des acteurs, sommant les habitants ou les usagers de participer à la délibération en tant que sujets, faisant fi des situations différenciées, des positions culturelles et sociales de chacun et du caractère non équitable des procédures, comme la coupure entre délibération et décision. Cette injonction, omniprésente sur notre terrain d'enquête, fait reposer le sort apparent des situations sur des conseillers qui ne les maîtrisent pourtant qu'à la marge. L'action publique s'appuie en effet le plus souvent sur le consentement. Elle vise à «faire bouger le sens commun [...] d'acteurs-auteurs [...] usagers modèles» en mettant à l'épreuve leur «sens de la légalité, [l']appréciation juste de [leurs] capacités, [la] prise en compte discursive de la pluralité des points de vue [et la] faculté de se couler dans les dispositifs» (Chateauraynaud et Trabal, 2005 : 118). Si l'approche en termes de reconnaissance a conduit la recherche à étayer ce concept de compétences, nous mesurons donc aujourd'hui la «vulnérabilité de l'homme capable» (Thévenot, 2007: 140). L'entreprise de capacitation menée par les chercheurs a certes légitimé des mobilisations citadines, mais elle a surtout contribué à la diffusion rapide d'une "responsabilisation » accrue des citoyens (Martuccelli, 2004: 479). Le plus souvent, cette « responsabilisation» de chacun ne repose sur aucun nouveau pouvoir, mais surtout celle-ci est censée être l'effectuation même de ce «pouvoir» distribué par les institutions. Un pouvoir sur soi, avant d'être un pouvoir sur le monde.

Pour discuter rapidement la place qu'occupe ce terme de compétence dans la sociologie pragmatiste, nous sommes parti de deux entrées, celle de la cognition distribuée (Conein, 2004) et celle de la vulnérabilité et du

cipation publique? Ces compétences en font-ils des acteurs naturels de la participation à Paris?» Questionnaire charte parisienne de la participation, Direction de la décentralisation et des relations avec les associations, les territoires et les citoyens, Mission démocratie locale. 
trouble (Breviglieri et Trom, 2003). Dans son approche du travail d'Erving Goffman, Joseph (1998) a toujours insisté sur la vulnérabilité des acteurs, afin de donner toute son importance à la notion d'accessibilité, seule à même de rendre lisible et de ménager cette vulnérabilité. Accompagnée par les réflexions initiales de Thévenot sur les questions du proche et de la familiarité (1994), la jeune équipe menée par Marc Breviglieri, Joan Stavo-Debauge et Luca Pattaroni a ensuite développé plus spécifiquement la question des publics vulnérables en remettant en cause l'exigence «considérable» et les « excès » de l'anthropologie capacitaire de la sociologie interactionniste pragmatiste (Breviglieri, 2008: 9), tout en inscrivant pourtant sa démarche dans un enjeu de reconnaissance des compétences «discrètes et familières » (ibid.: 15). Cet enchâssement de la question capacitaire dans les enjeux de lutte pour la reconnaissance (Honneth, 2000) oriente la réflexion critique sur la compétence comme opérateur de «partage des êtres » (Genard et Cantelli, 2008 : 4). Il n'interdit pas d'«abaisser» les exigences de l'anthropologie capacitaire propres à la sociologie pragmatiste et de «réviser les frontières de la différence anthropologique qu'elle affirme» entre la compétence des mobiles-mobilisés et l'incompétence des fixes-conformistes (Breviglieri, 2008: 19). Mais si cette attention au trouble, aux «foyers d'humiliation» (ibid.) et «aux espaces où se manifestent des luttes pour la reconnaissance des compétences» (Genard et Cantelli, 2008: 9) est indispensable, elle détourne la réflexion capacitaire d'un autre travail, celui portant sur la production situationnelle des compétences, en deçà de toute exigence politique et morale de reconnaissance. De nombreux auteurs relevant de ce même courant de recherche ${ }^{32}$ ont chacun à leur manière interrogé cette distribution en prenant au sérieux l'interobjectivité qui participe à cette construction sociale des compétences. Pour Francis Chateauraynaud, par exemple, il s'agit d'enquêter «dans les milieux d'activité où s'expose différemment la fabrique des prises sur le monde $»^{33}$ (2011: 461). Ce décentrement permet tout d'abord de sortir d'une conception dans laquelle ce sont les personnes qui portent les situations par leurs compétences critiques, leur travail d'enquête ou leur réflexivité pratique, pour considérer d'abord des situations contraignantes ou habilitantes. Une réunion de conseil de quartier ou une gestion de copropriété coopérative sont des situations qui portent des individus, toujours à la

32. Notamment: Bernard Conein (2004), Nicolas Dodier (1993), Michel Callon (1986), Bruno Latour (1994), Laurent Thévenot (2006) et Francis Chateauraynaud (2006).

33. Rappelons que, pour cet auteur, une compétence critique «renvoie à la faculté d'élaborer des prises pour agir sur un processus ou pour s'en forger une représentation adéquate» (Chateauraynaud, 2011: 21). S'il reprend ici une définition pragmatique assez classique du concept de compétence, la notion de prise s'apparente à celle d'affordance, développée dans les théories de l'action située à la suite de James Gibson (1979), qui désigne les prises particulières qui naissent de l'interaction entre des objets, des dispositifs et des situations qui convoquent l'action et des acteurs qui éprouvent et produisent ces opportunités pour tout à la fois les révéler et les incarner. 
fois potentiellement capables ET incapables (Genard et Cantelli, 2008: 7). Ensuite, ce décentrement permet de prendre en compte le fait que ce sont les relations ENTRE les «êtres» présents dans ces situations et leurs prises respectives qui déterminent l'issue de ces situations. Tirer les conséquences de l'importance des situations et des relations qui vont «prendre» (ou non) au sein de ces situations implique de prendre au sérieux l'incertitude plus ou moins grande qui caractérise ces relations aux situations, voire «l'inquiétude» qu'elles provoquent (Thévenot, 2006: 12). Par ailleurs, orienter le regard vers les situations plus que sur les capacités permet de ne pas faire reposer l'enjeu des mobilisations, et donc l'injonction capacitaire, sur un amont (expertise d'usage, savoir habitant, etc.), mais d'abord sur les prises qui sont éprouvées en situation lors de ces mobilisations.

À l'heure où les sciences sociales sont appelées à participer à l'élaboration et à la conduite des dispositifs participatifs, il ne s'agit plus seulement, nous semble-t-il, de mettre en cause une instrumentalisation de la recherche par une reprise de la critique caractéristique des modes de domination actuels, mais aussi de réinterroger la pertinence des concepts que nous utilisons pour éprouver à nouveaux frais ces situations de domination. Notre terrain nous a donné l'occasion de réinterroger celui de compétence, développé par les dispositifs participatifs et porté par la sociologie pragmatiste et la sociologie urbaine. Pour dépasser le face-à-face trompeur entre participation et représentation, nous nous sommes appuyé sur une expérience coopérative en faisant l'hypothèse que c'est moins le contenu critique de ce qui est dit au sein des enceintes participatives que l'élaboration d'un faire démocratique alternatif, notamment coopératif, qui met à l'épreuve d'ellemême la collectivité et les dispositifs institutionnels qu'elle se donne. Cette approche en termes de coopération permet de contourner l'injonction capacitaire, caractéristique des dispositifs de démocratie locale, pour mettre en avant l'émancipation critique que peut produire cette mise à l'épreuve publique d'un tel faire démocratique alternatif.

Cette démarche nous amène à reconsidérer les approches de la participation animées essentiellement par un objectif de reconnaissance des compétences des gouvernés par les institutions, pour développer une approche en termes d'émancipation (Boltanski, 2009) s'intéressant aux " cours d'action » (Joseph, 1998) qui mettent à l'épreuve les institutions et les gouvernants. Premièrement, cette approche donne alors toute sa place à la confrontation, non pas en termes de dissensus nécessaire à la délibération, mais en termes de faire problématique donnant aux préoccupations privées leurs dimensions publiques. Deuxièmement, considérer la confrontation comme un faire problématique conduit à prendre au sérieux la production des conditions coopératives de résolution de ce dernier, pour voir dans quelle mesure ces conditions coopératives sont susceptibles de forger une émancipation critique des participants. 
On pourrait alors imaginer des dispositifs publics contribuant à la démocratie locale qui reposent sur cet objectif d'émancipation. L'effectivité de ces dispositifs reposerait alors sur leur faculté à produire les conditions coopératives d'émancipation critique des participants vis-à-vis des dispositifs eux-mêmes, pour mieux agir sur ces situations problématiques. Il nous semble donc que ce passage d'une compétence participative à une émancipation coopérative permet à la fois de surmonter l'instrumentalisation capacitaire et de mieux travailler l'articulation entre la constitution des prises et la portée critique des processus d'émancipation.

\section{Bibliographie}

Bächtiger, André, Markus Spörndli, Jurg Steiner et Marco Steenbergen, 2004, Deliberative Politics in Action. Analysing Parliamentary Discourse, Cambridge, Cambridge University Press.

Bacqué, Marie-Hélène et Yves Sintomer (sous la dir. de), 2011, La démocratie participative. Histoires et généalogies, Paris, La Découverte.

Berelson, Bernard, 1952, Content Analysis in Communication Research, New York, Free Press.

Berger, Mathieu, 2009, «Quand pourrons-nous parler des choses? Quelques contraintes à la référentialité des voix profanes dans un dispositif d'urbanisme participatif», dans Fabrizio Cantelli, Luca Pattaroni, Marta Roca i Escoda et Joan Stavo-Debauge (sous la dir. de), Sensibilités pragmatiques. Enquêter sur l'action publique, Bruxelles, Peter Lang, p. 253-280.

Berry-Chikhaoui, Isabelle et Agnès Deboulet, 2000, Les compétences des citadins dans le Monde arabe: Penser, faire et transformer la ville, Paris, Karthala.

Bessy, Christian et Francis Chateauraynaud, 1995, Experts et faussaires, Pour une sociologie de la perception, Paris, Métailié.

Bevort, Antoine et Michel Lallement (sous la dir. de), 2006, Le capital social, performance, équité et réciprocité, Paris, La Découverte.

Blondiaux, Loïc et Yves Sintomer, 2002, «L'impératif délibératif», Politix, vol. 15, $\mathrm{n}^{\circ} 57, \mathrm{p} .17-35$.

Blondiaux, Loïc, 2007, «Faut-il se débarrasser de la notion de compétence politique? Retour critique sur un concept classique de la science politique», Revue française de science politique, vol. $57, \mathrm{n}^{\circ} 6$, p. 759-774.

Blondiaux, Loïc, 2008, «Démocratie délibérative $v s$. Démocratie agonistique. Le statut du conflit dans les pratiques de participation contemporaines », Raisons politiques, vol. 2, n ${ }^{\circ} 30$, p. 131-147.

Bohman, James, 2008, «Réaliser la démocratie délibérative comme mode d'enquête: le pragmatisme, les faits sociaux et la théorie normative» [traduit par Charles Girard], Tracés. Revue de sciences humaines, ${ }^{\circ} 15$, Pragmatismes, consulté sur Internet (http://traces.revues.org/index883.html) le 3 mai 2009.

Boltanski, Luc, 1990, L'amour et la justice comme compétences; Trois essais de sociologie de l'action, Paris, Métailié.

Boltanski, Luc, 2008a, «Institutions et critique sociale. Une approche pragmatique de la domination ", Tracés. Revue de sciences humaines, n ${ }^{\circ}$, Hors-série 2008. Présent et futurs de la critique, consulté sur Internet (http://traces.revues.org/ index2333.html) le 22 octobre 2009.

Boltanski, Luc, 2008b, Rendre la réalité inacceptable, Paris, Demopolis. 
Boltanski, Luc, 2009, De la critique. Précis de sociologie de l'émancipation, Paris, Gallimard.

Boltanski, Luc, Yann Darré et Marie-Ange Schiltz, 1984, «La dénonciation », Actes de la recherche en sciences sociales, $\mathrm{n}^{\circ}$ 51, p. 3-40.

Boltanski, Luc et Laurent Thévenot, 1991, De la justification, les économies de la grandeur, Paris, Gallimard.

Bonny, Yves, Sylvie Ollitraut, Yvon Le Caro et Régis Keerle, 2012, Espaces de vie, espaces enjeux: entre investissements ordinaires et mobilisations politiques, Rennes, Presses universitaires de Rennes.

Breviglieri, Marc, 2008, «Penser la dignité sans parler le langage de la capacité à agir ", dans Jean-Paul Payet et Alain Battegay (sous la dir. de), La reconnaissance à l'épreuve. Explorations socio-anthropologiques, Lille, Presses universitaires du Septentrion, p. 83-92. [Les pages citées dans le texte correspondent à une version de travail sensiblement plus longue.]

Breviglieri, Marc et Danny Trom, 2003, «Troubles et tensions en milieu urbain. Les épreuves citadines et habitantes de la ville», dans Daniel Cefaï et Dominique Pasquier (sous la dir. de), Les sens du public. Publics politiques, publics médiatiques, Paris, Presses universitaires de France, p. 399-416.

Buravoy, Michael, 2010, « Revisiter les terrains. Esquisse d'une ethnographie réflexive», dans Daniel Cefaï (sous la dir. de), L'engagement ethnographique, Paris, Éditions de l'École des hautes études en sciences sociales (EHESS), p. 295-354.

Busquet, Grégory, Claire Carriou, Amélie Flamand et Héloïse Nez, 2010, «La démocratie participative à Paris sous le premier mandat Bertrand Delanoë (2001-2008): une 'nouvelle ère démocratique'?», dans Marie-Hélène Bacqué et Yves Sintomer (sous la dir. de), La démocratie participative inachevée. Genèse, adaptations et diffusions, Gap, Yves Michel/ADELS, p. 137-155.

Callon, Michel, 1986, «Éléments pour une sociologie de la traduction. La domestication des coquilles Saint-Jacques et des marins-pêcheurs dans la baie de Saint-Brieuc», L'année sociologique, vol. 36, p. 169-208.

Cantelli, Fabrizio et Jean-Louis Genard, 2007, Action publique et subjectivité, Paris, Droit et société, Librairie générale de droit et de jurisprudence (LGDJ), nº 46.

Castells, Manuel, 1975, Luttes urbaines et pouvoir politique, Paris, Maspero.

Castoriadis, Cornélius, 1999 [1975], L’institution imaginaire de la société, Paris, Seuil.

Cefaï, Daniel (sous la dir. de), 2010, L'engagement ethnographique, Paris, Éditions de l'École des hautes études en sciences sociales (EHESS).

Centre d'analyse stratégique, 2008, La participation des citoyens et l'action publique, Agence COM\&O, Paris, La Documentation française, mai.

Chateauraynaud, Francis, 2006, Les asymétries de prises. Des formes de pouvoir dans un monde en réseaux, document de travail du Groupe de sociologie pragmatique et réflexive (GSPR), consulté sur Internet (http://gspr.ehess.free.fr/ documents/FC-asymetries-de-prises-2006.pdf) le 12 octobre 2010.

Chateauraynaud, Francis, 2011, Argumenter dans un champ de forces. Essai de balistique sociologique, Paris, Petra.

Chateauraynaud, Francis et Patrick Trabal, 2005, Conducteurs ordinaires, et extraordinaires, aux prises avec les dispositifs publics. Sociologie des expériences routières et de leurs mises en forme argumentatives, Rapport final, Paris, Association DOXA, PREDIT 3 2002-2006, Groupe opérationnel n 3 (Nouvelles connaissances pour la sécurité).

Chauvière, Michel, 2007, Trop de gestion tue le social. Essai sur une discrète chalandisation, Paris, La Découverte. 
Clot, Yves, 1995, Le travail sans l'homme?, Paris, La Découverte.

Conein, Bernard, 2004, «Cognition distribuée, groupe social et technologie cognitive», Réseaux, vol. 2, nº 124, p. 53-79.

Conein, Bernard et Eric Jacopin, 1994, «Action située et cognition», Revue française de sociologie, vol. 36, $\mathrm{n}^{\circ} 4$, p. 475-499.

Coutant, Isabelle, 2000, Politiques du squat. Scènes de la vie d'un quartier populaire, Paris, La Dispute.

de Certeau, Michel, 1990, L'invention du quotidien, t. 1 L'art de faire, Paris, Gallimard.

De Munck, Jean et Bénédicte Zimmermann (sous la dir. de), 2008, La liberté au prisme des capacités. Amartya Sen au-delà du libéralisme, Paris, Éditions de l'École des hautes études en sciences sociales (EHESS).

Dewey, John, 1938, Experience and Education, New York, Collier Books.

Dodier, Nicolas, 1993, «Les appuis conventionnels de l'action. Éléments d'une pragmatique sociologique », Réseaux, n 62, p. 63-85.

Dodier, Nicolas, 2001, «Une éthique radicale de l'indexicalité», dans Michel de Fornel, Albert Ogien et Louis Quéré (sous la dir. de), L'ethnométhodologie. Une sociologie radicale, Paris, La Découverte, p. 315-330.

Dodier, Nicolas et Isabelle Baszanger, 1997, «Totalisation et altérité dans l'enquête ethnographique», Revue française de sociologie, vol. 38, n 1, p. 37-66.

Forsé, Michel et Claudette Guibet Lafaye, 2008, «La démocratie participative et les devoirs du citoyen ", Archives européennes de sociologie, p. 173-204.

Fourniau, Jean-Michel, 2007, «L'expérience démocratique des 'citoyens en tant que riverains' dans les conflits d'aménagement», Revue européenne des sciences sociales, t. XLV, n ${ }^{\circ} 136$, p. 149-179.

Genard, Jean-Louis, 1999, La grammaire de la responsabilité, Paris, Cerf.

Genard, Jean-Louis et Fabrizio Cantelli, 2008, «Êtres capables et compétents : lecture anthropologique et pistes pragmatiques», Sociologies, «Théories et recherches », mis en ligne le 27 avril 2008, consulté sur Internet (http:// sociologies.revues.org/index1943.html) le 30 septembre 2009.

Gibson, James, 1979, The Ecological Approach to Visual Perception, Boston, Houghton Mifflin.

Giddens, Anthony, 2000, Les conséquences de la modernité, Paris, L'Harmattan.

Honneth, Axel, 2000, La lutte pour la reconnaissance, Paris, Cerf.

Jacobs, Jane, 1961, The Death and Life of Great American Cities, New York, Vintage.

Jobert, Arthur, 1998, «L'aménagement en politique ou ce que le syndrome NIMBY nous dit de l'intérêt général», Politix, n 42, p. 67-92.

Joseph, Isaac, 1996, «Les compétences de rassemblement. Une ethnographie des lieux publics », Enquête, n 4, p. 107-122.

Joseph, Isaac, 1998, Erving Goffman et la microsociologie, Paris, Presses universitaires de France.

Lagroye, Jacques, 1991, Sociologie politique, Paris, Presses de la Fondation nationale des sciences politiques, Dalloz.

Latour, Bruno, 1994, «Une sociologie sans objet? Remarques sur l'interobjectivité», Sociologie du travail, p. 587-606.

Latour, Bruno, 1995, Le métier de chercheur. Regard d'un anthropologue, Paris, Institut national de la recherche agronomique (INRA).

Lefebvre, Henri, 1968, Le droit à la ville, Paris, Seuil.

Linhardt, Dominique et Cédric Moreau de Bellaing, 2005, «Légitime violence? Enquêtes sur la réalité de l'État démocratique», Revue française de science politique, vol. $55, \mathrm{n}^{\circ} 2$, p. $269-298$. 
Martuccelli, Danilo, 2004, «Figures de la domination», Revue française de sociologie, vol. $45, n^{\circ} 3$, p. 469-497.

Nez, Héloïse, 2007, «Les conseils de quartier à Paris: de l'expérimentation à l'institutionnalisation", Rapport de recherche, dans Les dispositifs participatifs locaux en Île-de-France et en Europe: Vers une démocratie technique?, Programme de recherche Partenariats institutions citoyens pour la recherche et l'innovation (PICRI) / Île-de-France.

Olivier de Sardan, Jean-Pierre, 1995, «La politique du terrain. Sur la production des données en anthropologie», Enquête, n ${ }^{\circ} 1$, p. 71-112.

Quéré, Louis, 2009, «Intérêt et limites de la théorie des régimes pragmatiques pour la sociologie de l'action», dans Marc Breviglieri, Claudette Lafaye et Dany Trom (sous la dir. de), Compétences critiques et sens de la justice, Paris, Economica.

Raymond, Henri, 1984, L'architecture, les aventures spatiales de la raison, Paris, Centre de création industrielle (CCI)-Centre Georges Pompidou.

Rosanvallon, Pierre, 2006, La contre-démocratie. La politique à l'âge de la défiance, Paris, Seuil.

Sintomer, Yves, 2007, Le pouvoir au peuple. Jurys citoyens, tirage au sort et démocratie participative, Paris, La Découverte.

Stavo-Debauge, Joan, 2011, «De la critique, une critique. Sur le geste 'radical' de Luc Boltanski», EspacesTemps.net, consulté sur Internet (http://espacestemps.net/ document8658.html), le 18 septembre 2011.

Thévenot, Laurent, 1990, «L'action qui convient», Les formes de l'action collective (série «Raisons Pratiques», n 1 ), Paris, Éditions de l'École des hautes études en sciences sociales (EHESS).

Thévenot, Laurent, 1994, «Le régime de familiarité, des choses en personne», Genèses, ${ }^{\circ}$ 17, p. 72-101.

Thévenot, Laurent, 2006, L'action au pluriel. Sociologie des régimes d'engagement, Paris, La Découverte.

Thévenot, Laurent, 2007, «Reconnaissances avec Paul Ricœur et Axel Honneth », dans Christian Delacroix, François Dosse et Patrick Garcia (sous la dir. de), Paul Ricour et les sciences humaines, Paris, La Découverte.

Trom, Danny, 1997, «Voir le paysage, enquêter sur le temps. Narration du temps historique, engagement dans l'action et rapport visuel au monde», Politix, vol. 10, $\mathrm{n}^{\circ}$ 39, p. 86-108.

Trom, Danny, 1999, «De la réfutation de l'effet NIMBY considérée comme une pratique militante», Revue française de science politique, Fondation nationale des sciences politiques, vol. $49, \mathrm{n}^{\circ} 1, \mathrm{p} .31-50$.

Vandenberghe, Frédéric, 2009, «Structures, grandeurs et platitudes dans la nouvelle sociologie française», dans Marc Breviglieri, Claudette Lafaye et Dany Trom, Compétences critiques et sens de la justice, Paris, Economica, p. 375-387.

Wekerlé, Gerda, 1999, «De la coveillance à la ville sûre», Les Annales de la recherche urbaine, ${ }^{\circ}$ 83-84, p. 164-169.

Wybo, Gilles, Annabel Benhaeim et Catherine Feldman, 2008, «Savoir-être, la compétence qui monte», Courrier cadres.com, consulté sur Internet (http:// sufco.phpnet.org/scuio/pmb/opac_css/index.php?lvl=notice_display\&id=3234) le 28 janvier 2010. 